\title{
Detection of Retinal Vascular Bifurcations by Trainable V4-Like Filters
}

\author{
George Azzopardi and Nicolai Petkov \\ Johann Bernoulli Institute for Mathematics and Computer Science, \\ University of Groningen, The Netherlands \\ $\{$ g.azzopardi, n.petkov\}@rug.nl
}

\begin{abstract}
The detection of vascular bifurcations in retinal fundus images is important for finding signs of various cardiovascular diseases. We propose a novel method to detect such bifurcations. Our method is implemented in trainable filters that mimic the properties of shape-selective neurons in area V4 of visual cortex. Such a filter is configured by combining given channels of a bank of Gabor filters in an AND-gate-like operation. Their selection is determined by the automatic analysis of a bifurcation feature that is specified by the user from a training image. Consequently, the filter responds to the same and similar bifurcations. With only 25 filters we achieved a correct detection rate of $98.52 \%$ at a precision rate of $95.19 \%$ on a set of 40 binary fundus images, containing more than 5000 bifurcations. In principle, all vascular bifurcations can be detected if a sufficient number of filters are configured and used.
\end{abstract}

Keywords: DRIVE, Gabor filters, retinal fundus, trainable filters, V4 neurons, vessel bifurcation.

\section{Introduction}

The vascular topographical geometry in the retina is known to conform to structural principles that are related to certain physical properties [14. The analysis of the geometrical structure is very important as deviations from the optimal principles may indicate some cardiovascular diseases, such as hypertension 17. and atherosclerosis [4]; a comprehensive analysis is given in [12]. The identification of vascular bifurcations is one of the basic steps in this analysis.

More than 100 vascular bifurcations can be seen in a typical retinal fundus image. Their manual detection by a human observer is a tedious and time consuming process. The existing attempts to automate the detection of retinal vascular bifurcations can be categorized into two classes usually referred to as geometrical-feature based and model based approaches. The former involve extensive preprocessing such as segmentation and skeletonization followed by local pixel processing and branch point analysis. These techniques are known for their robustness in bifurcation localization 22315/8. On the other hand, model based approaches are usually more adaptive and have smaller computational complexity which makes them more appropriate for real-time applications [116].

A. Berciano et al. (Eds.): CAIP 2011, LNCS 6854, pp. 451-459 2011.

(C) Springer-Verlag Berlin Heidelberg 2011 
However, model based approaches are known to suffer from insufficient generalization ability as they are usually unable to model all the features of interest. Consequently, these methods may fail to detect some relevant features.

In this paper we propose trainable filters for the detection of vascular bifurcations in retinal fundus images. Our approach requires a single-step training process where an observer specifies a typical bifurcation by a point of interest in an image. The specified feature is then used to automatically configure a bifurcation detector by determining the properties of all line segments in the concerned feature and their mutual geometrical arrangement. This training procedure can be repeated as many times as required in order to configure a number of filters based on different specified features of interest. The filters can then be applied on retinal fundus images to detect the features that are similar to the patterns that were used to configure the filters.

The rest of the paper is organized as follows: In Section 2 we present our method and demonstrate how it can be used to detect retinal vascular bifurcations. In Section 3, we apply the proposed nonlinear filters on retinal fundus images from the DRIVE dataset [15]. Section 4 contains a discussion and conclusions.

\section{Proposed Method}

\subsection{Overview}

Fig [1 a shows a bifurcation encircled in a binarized retinal fundus image from the DRIVE dataset [15. Such a feature, which is shown enlarged in Fig [1 b, is used to automatically configure a detector that will respond to the same and similar patterns.

Each of the three ellipses shown in Fig 10 represents the support or receptive field (RF) of a sub-unit that detects a line of a given orientation and width, while the central circle represents the RF of a group of such sub-units. The response of the proposed bifurcation detector is computed by combining the responses of the concerned sub-units by multiplication. The preferred orientations of the subunits and the mutual spatial arrangement of their RFs are determined by the local pattern used for the configuration of the concerned filter. Consequently,

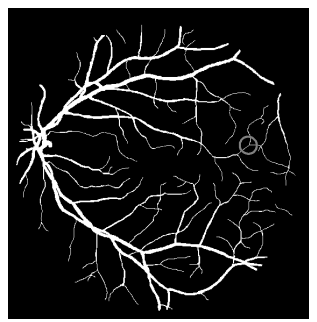

(a)

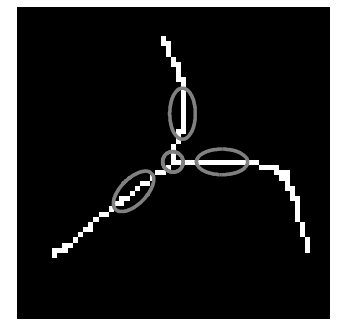

(b)

Fig. 1. (a) The circle indicates a bifurcation that is selected by a user. (b) Enlargement of the selected feature. The ellipses represent the support of line detectors that are identified as relevant for the concerned feature. 
that filter is selective for the presented local combination of lines of specific orientations and widths.

Such a design is inspired by electrophysiological evidence that some neurons in area V4 of visual cortex are selective for moderately complex stimuli, such as curvatures, that receive inputs from a group of orientation-selective cells in areas V1 and V2 9]10]11. Moreover, there is psychophysical evidence [6] that curve contour parts are likely detected by an AND-gate-like operation that combines the responses of afferent orientation-selective sub-units by multiplication. An AND-gate-like model produces a response only when all its afferent sub-units are stimulated; i.e. all constituent parts of a stimulus are present.

In the next sub-sections, we explain the automatic configuration process of a bifurcation detector. The configuration process determines which responses of which Gabor filters in which locations need to be multiplied in order to obtain the output of the filter.

\subsection{Orientation-Selective Sub-units Based on Gabor Filters}

The input to the orientation-selective sub-units mentioned above is provided by two-dimensional (2D) Gabor filters, which are established models of V1/V2 cells. We denote by $g_{\lambda, \theta}(x, y)$ the half-wave rectified response of a Gabor filter of preferred wavelength $\lambda$ and orientation $\theta$ to a given input image. Such a filter has also other parameters, namely spatial aspect ratio, bandwidth and phase offset, that we skip here for brevity. We set their values as proposed in [13.

Since we work in a multiscale setting, we re-normalize all Gabor functions that we use in such a way that all positive values of such a function sum up to 1 while all negative values sum up to -1 . We use symmetric Gabor functions as they respond to line structures and we are interested to detect the presence of vessels in retinal fundus images.

We use a bank of Gabor filters with 5 wavelengths $(\Lambda=\{4,4 \sqrt{2}, 8,8 \sqrt{2}, 16\})$ and 8 equidistant orientations $\left(\Theta=\left\{0, \frac{\pi}{8}, \ldots, \frac{7 \pi}{8}\right\}\right)$ that we apply on images of size $565 \times 584$. In such images, the blood vessels have widths of 1 to 7 pixels. Fig $2 \mathrm{a}$ illustrates the maximum value superposition of the thresholded responses of the concerned bank of Gabor filters obtained for the bifurcation image shown in Fig.1b. All responses are thresholded at a given fraction $t_{1}=0.2$ of the maximum response of $g_{\lambda, \theta}(x, y)$ across all combinations of values $(\lambda, \theta)$ used and all positions $(x, y)$ in the image.

\subsection{Sub-unit Parameters}

A sub-unit uses as inputs the responses of a certain Gabor filter characterized by the parameter values $(\lambda, \theta)$ around a certain position $(\rho, \phi)$ with respect to the center of the filter. A sub-unit is thus characterized by four parameters: $(\lambda, \theta, \rho, \phi)$. The values of such parameters for a sub-unit are obtained as follows.

We consider the responses of the bank of Gabor filters along a circle of a given radius $\rho$ around the selected point of interest (Fig,2). In each position along that circle, we take the maximum of all responses across the possible values of $(\lambda, \theta)$. 
If this value is greater than the corresponding values for the neighboring positions along an arc of angle $\frac{\pi}{8}$ the concerned position is chosen as a center of the RF of a sub-unit. Its coordinates $(\rho, \phi)$ are determined with respect to the center of the filter. The pair of values $(\lambda, \theta)$ for which the concerned local maximum is reached are the preferred wavelength and orientation of the sub-unit.

In our experiments, we configure bifurcation detectors using multiple values of the parameter $\rho$. For non-zero values of $\rho$ we determine a group of sub-units with the method mentioned above. For $\rho=0$, we consider the responses of the bank of Gabor filters used at the specified point of interest. For such a location, we consider all combinations of $(\lambda, \theta)$ for which the corresponding responses $g_{\lambda, \theta}(x, y)$ are greater than a fraction $t_{2}=0.75$ of the maximum of $g_{\lambda, \theta}(x, y)$ across the different combinations of values $(\lambda, \theta)$ used. For each value $\theta$ that satisfies such a condition, we consider a single value of $\lambda$, the one for which $g_{\lambda, \theta}(x, y)$ is the maximum of all responses across all values of $\lambda$. At this central location, multiple sub-units can thus be defined and their RFs are centered at the same position with polar coordinates $(\rho=0, \phi=0)$.

We denote the set of parameter value combinations, which fulfill the above conditions, by $S_{f}=\{(\lambda, \theta, \rho, \phi)\}$. The subscript $f$ stands for the local pattern around the selected point of interest. Every tuple in the set $S_{f}$ specifies the parameters of a sub-unit.

For the point of interest shown in Fig $2 \mathrm{a}$ and two given values of the radius $\rho(\{0,10\})$, the selection method described above results in five sub-units with parameter values specified by the tuples in the following set; $S_{f}=\{(\lambda=4, \theta=$ $0, \rho=0, \phi=0),\left(\lambda=4, \theta=\frac{\pi}{2}, \rho=0, \phi=0\right),(\lambda=4, \theta=0, \rho=10, \phi=$ $\left.1.34),\left(\lambda=4, \theta=\frac{3 \pi}{4}, \rho=10, \phi=3.75\right),\left(\lambda=4, \theta=\frac{\pi}{2}, \rho=10, \phi=6.27\right)\right\}$. The last tuple in that list, $\left(\lambda=4, \theta=\frac{\pi}{2}, \rho=10, \phi=6.27\right)$, for instance, describes a sub-unit that collects its inputs from the responses of a Gabor filter with $\lambda=4$ and $\theta=\frac{\pi}{2}$, i.e. a Gabor filter that strongly responds to horizontal lines $\left(\theta=\frac{\pi}{2}\right)$

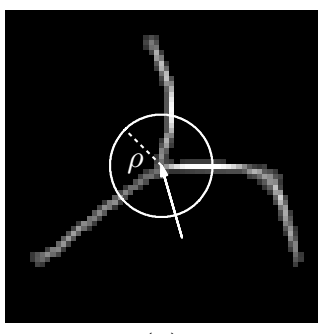

(a)

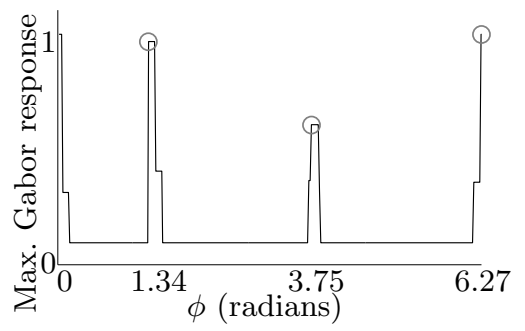

(b)

Fig. 2. (a) The gray-level intensity of every pixel is the maximum value superposition of the thresholded responses from a bank of Gabor filters at that position; $\max _{\lambda \in \Lambda, \theta \in \Theta}\left|g_{\lambda, \theta}(x, y)\right|_{t_{1}}$. The arrow indicates the location of the point of interest selected by a user, while the bright circle of a given radius $\rho$ indicates the considered locations. (b) Values of the maximum value superposition of Gabor filter responses along the concerned circle of radius $\rho=10$ around the point of interest. The marked local maxima are caused by the three blood vessels. 
of width of $\left(\frac{\lambda}{2}=\right) 2$ pixels, around a position of $(\rho=) 10$ pixels to the right $(\phi=6.27)$ of the center of the filter. This selection is the result of the presence of a horizontal vessel to the right of the center of the feature that is used for the configuration of the filter.

\subsection{Sub-unit Response}

We denote by $s_{\lambda, \theta, \rho, \phi}(x, y)$ the response of a sub-unit, which we compute as follows. We consider the responses $g_{\lambda, \theta}(x, y)$ of a Gabor filter with preferred wavelength $\lambda$ and orientation $\theta$ around position $(\rho, \phi)$ with respect to the center of the filter. We weight these responses by a $2 \mathrm{D}$ Gaussian function with a standard deviation that is a linear function of parameter $\rho$. We define the output of the sub-unit as the maximum value of all the weighted responses of the concerned Gabor filter. This result is shifted by $\rho$ in the direction opposite to $\phi$.

Fig.3 illustrates the computation of the responses of three sub-units. Each of the three bright blobs shown is an intensity map of a 2D Gaussian function mentioned above. The three ellipses illustrate the orientations and wavelengths of the corresponding Gabor filters. The responses $g_{\lambda, \theta}(x, y)$ of such a filter are weighted by the respective $2 \mathrm{D}$ Gaussian function and the maximum result is shifted by the corresponding vector.

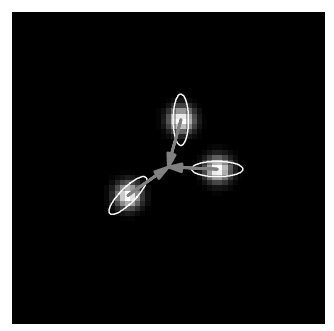

Fig. 3. Computation of sub-unit responses. The three bright blobs are intensity maps for 2D Gaussian functions that model the corresponding sub-unit RFs. The three ellipses illustrate the orientations and wavelengths of the corresponding Gabor filters. A sub-unit response is computed as the maximum value of the weighted responses of such a Gabor filter with the respective 2D Gaussian function. The result is shifted by the corresponding vector.

\subsection{Filter Response}

We define a nonlinear filter with output $r_{S_{f}}$ as the geometric mean of all quantities $s_{\lambda, \theta, \rho, \phi}(x, y)$ that belong to the specific selection determined by $S_{f}$ :

$$
r_{S_{f}}(x, y)=\left|\left(\prod_{(\lambda, \theta, \rho, \phi) \in S_{f}} s_{\lambda, \theta, \rho, \phi}(x, y)\right)^{\frac{1}{\mid S_{f}} \mid}\right|_{t_{3}}
$$


where $|\cdot|_{t_{3}}$ stands for thresholding the response at a fraction $t_{3}$ of its maximum.

Rotation invariance is achieved by manipulating the set of parameter values in $S_{f}$, rather than by computing them from the responses to a rotated version of the original pattern. Using the set $S_{f}$ that defines the concerned filter, we can form a new set $\Re_{\psi}\left(S_{f}\right)=\left\{(\lambda, \theta+\psi, \rho, \phi+\psi) \mid(\lambda, \theta, \rho, \phi) \in S_{f}\right\}$. The rotation invariant response is then defined as $\widehat{r}_{S_{f}}(x, y)=\max _{\psi}\left(r_{\Re_{\psi}\left(S_{f}\right)}(x, y)\right)$.

\section{$3 \quad$ Experimental Results}

We use the bifurcation illustrated in Fig 1 to configure a filter denoted by $S_{f_{1}}$

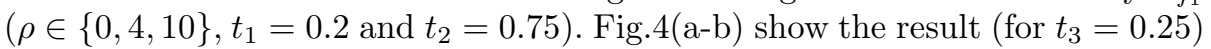
of the application of filter $S_{f_{1}}$ to the binary retinal fundus image shown in Fig 1 . The encircled regions are centered on the local maxima of the filter response and if two such regions overlap by $75 \%$, only the one with the stronger response is shown. Besides the original bifurcation, the filter successfully detects 5 other bifurcations with similar vessel orientations.

If the same filter is applied in a rotation invariant mode, a total of 38 similar features are detected, Fig 4 (c-d). This illustrates the strong generalization capability of this approach because $35.51 \%$ (38 out of 107) of the features of interest are detected by a single filter. Notable is the fact that this is achieved at a precision rate of $100 \%$, as the filter does not give any false positive responses. The threshold parameter $t_{3}$ can be used to tune the degree of generalization.

As to the remaining features that are not detected by this filter, we proceed as follows: we take one of these features that we denote by $f_{2}$ (Fig. (5) and train a second nonlinear filter, $S_{f_{2}}$, using it. With this second filter we detect 46 features of interest of which 20 coincide with features detected by filter $S_{f_{1}}$ and 26 are newly detected features. Merging the responses of the two filters results in the detection of 64 distinct features. We continue adding filters that are configured using features that have not been detected by the previously trained filters. A set of 10 filters that correspond to the features shown in Fig 5 proves sufficient to detect all 107 features of interest in the concerned image. A fixed response

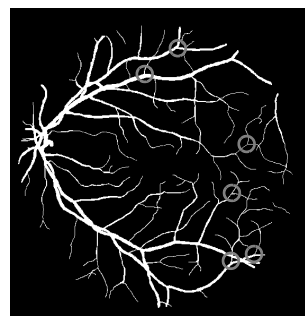

(a)

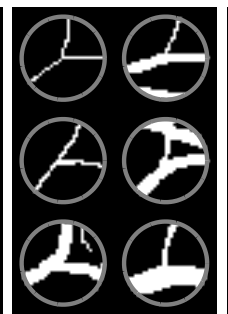

(b)

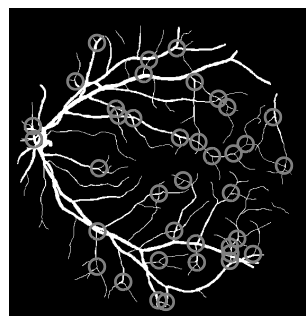

(c)

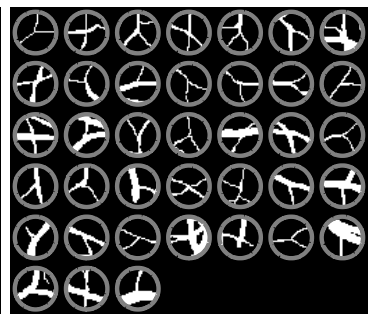

(d)

Fig. 4. (a) Result of applying the filter $S_{f_{1}}$ in rotation non-invariant mode and (b) enlargements of the detected features given in descending order (left-to-right, top-tobottom) of the filter response. (c) Result of applying the filter in a rotation invariant mode and (d) enlargements of the detected features. 

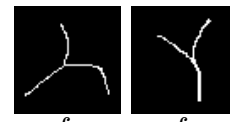

$f_{2}$

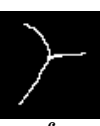

$f_{3}$

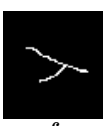

$f_{4}$

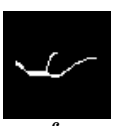

$f_{5}$

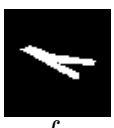

$f_{6}$

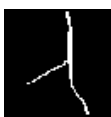

$f_{7}$

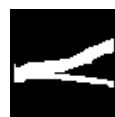

$f_{8}$

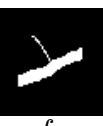

$f_{9}$

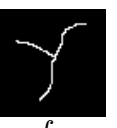

$f_{10}$

Fig. 5. A set of 10 bifurcations extracted from the image in Fig 1a, used to configure 10 filters

threshold of $t_{3}=0.25$ is applied for all filters. An important aspect of this result is that a recall rate of $100 \%$ is achieved at a precision rate of $100 \% 1$.

We apply these 10 filters on a larger dataset (DRIVE) of 40 binary retinal fundus images 2 . The ground truth of correct bifurcations was defined by the authors of this paper. For this larger dataset we achieve a recall rate $R$ of $97.3 \%$ and a precision rate $P$ of $94.71 \%$. We carried out further experiments by configuring up to 40 filters and varying the threshold parameter $t_{3}$ between 0.2 and 0.3 . We achieve optimal results for 25 filters and show them together with the results for 10 filters in Fig 6 . With 25 filters, the harmonic mean $(2 P R /(P+R))$ of the precison and recall reaches maximum at a recall rate of $98.52 \%$ and a precision rate of $95.19 \%$ for $t_{3}=0.28$.

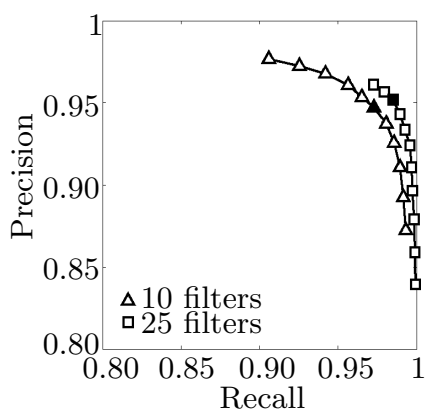

Fig. 6. Precision-recall plots obtained with 10 and 25 filters. For each plot the threshold parameter $t_{3}$ is varied between 0.2 and 0.3 . The precision rate increases and the recall rate decreases with an increasing value of $t_{3}$. The harmonic mean of precision and recall reaches a maximum at $R=0.9852$ and $P=0.9519$ for 25 filters and at $R=0.973$ and $P=0.9471$ for 10 filters. These points are marked by a filled-in square and triangle, respectively.

\section{Discussion and Conclusion}

We propose a novel approach for the automation of vascular bifurcation detection in retinal fundus images. Our proposed method is implemented in filters that simulate the properties of shape-selective V4 neurons in visual cortex.

\footnotetext{
${ }^{1}$ Recall rate is the percentage of true bifurcations that are successfully detected. Precision rate is the percentage of correct bifurcations from all detected features.

${ }^{2}$ Named in DRIVE 01_manual1.gif, 02_manual1.gif, ..., 40_manual1.gif
} 
The proposed V4-like filters are trainable, in that the structure of the filter is determined by a feature that is specified by a user. The way this is achieved is not by template matching, but rather by the extraction of information about the dominant orientations in the concerned feature and their mutual spatial arrangement. While such a filter reacts most strongly to the feature that was used to configure it, the filter also reacts to features which differ in the orientations of the involved line segments to a certain extent. The degree of generalization can be tuned by proper selection of the filter parameters. The automatic configuration of the proposed filters gives an edge to our approach over model based approaches regarding generalization ability.

Although one can find methods for local image feature analysis by combining filter responses at different scales (e.g. SIFT features [7]), to the best of our knowledge, the proposed approach is the first one which combines the responses of orientation-selective filters with their main area of support outside the point of interest.

In our experiments, we use a set of 40 binary retinal images provided as ground truth in the DRIVE dataset [15. In total, these images contain 5118 vessel bifurcations. We achieved a recall rate of $98.52 \%$ and a precision rate of $95.19 \%$ with the application of only 25 filters. The precision rate can be improved by performing additional analysis of the features that are detected by the filters. In [2] a recall rate of $95.82 \%$ was reported on a small dataset of five retinal images.

In principle, all vessel bifurcations can be detected if a sufficient number of filters are configured and used. The recall rate of $98.52 \%$ that we achieve means that on average only one to two out of 100 bifurcations are missed in a typical image. This is sufficient to the needs of the medical application at hand. We conclude that the proposed trainable filters are an effective means to automatically detect bifurcations in retinal vascular images.

\section{References}

1. Ali, C., Hong, S., Turner, J., Tanenbaum, H., Roysam, B.: Rapid automated tracing and feature extraction from retinal fundus images using direct exploratory algorithms. IEEE Transactions on Information Technology in Biomedicine 3, 125-138 (1999)

2. Bhuiyan, A., Nath, B., Chua, J., Ramamohanarao, K.: Automatic detection of vascular bifurcations and crossovers from color retinal fundus images. In: Third International IEEE Conference on Signal-Image Technologies and Internet-Based System (SITIS), pp. 711-718 (2007)

3. Chanwimaluang, T., Guoliang, F.: An efficient blood vessel detection algorithm for retinal images using local entropy thresholding. In: Proceedings of the 2003 IEEE International Symposium on Circuits and Systems (Cat. No.03CH37430) 5

4. Chapman, N., Dell'omo, G., Sartini, M., Witt, N., Hughes, A., Thom, S., Pedrinelli, R.: Peripheral vascular disease is associated with abnormal arteriolar diameter relationships at bifurcations in the human retina. Clinical Science 103 (2002)

5. Eunhwa, J., Kyungho, H.: Automatic retinal vasculature structure tracing and vascular landmark extraction from human eye image. In: International Conference on Hybrid Information Technology, vol. 7 (2006) 
6. Gheorghiu, E., Kingdom, F.: Multiplication in curvature processing. Journal of Vision 9 (2009)

7. Lowe, D.: Distinctive image features from scale-invariant keypoints. International Journal of Computer Vision 60, 91-110 (2004)

8. Martinez-Perez, M., Hughes, A., Stanton, A., Thom, S., Chapman, N., Bharath, A., Parker, K.: Retinal vascular tree morphology: A semi-automatic quantification. IEEE Transactions on Biomedical Engineering 49, 912-917 (2002)

9. Pasupathy, A., Connor, C.: Responses to contour features in macaque area $v 4$. Journal of Neurophysiology 82, 2490-2502 (1999)

10. Pasupathy, A., Connor, C.: Shape representation in area v4: Position-specific tuning for boundary conformation. Journal of Neurophysiology 86, 2505-2519 (2001)

11. Pasupathy, A., Connor, C.: Population coding of shape in area v4. Nature Neuroscience 5, 1332-1338 (2002)

12. Patton, N., Aslam, T., MacGillivray, T., Deary, I., Dhillon, B., Eikelboom, R., Yogesan, K., Constable, I.: Retinal image analysis: Concepts, applications and potential. Progress in Retinal and Eye Research 25, 99-127 (2006)

13. Petkov, N.: Biologically motivated computationally intensive approaches to image pattern-recognition. Future Generation Computer Systems 11, 451-465 (1995)

14. Sherman, T.: On connecting large vessels to small - the meaning of murray law. Journal of General Physiology 78, 431-453 (1981)

15. Staal, J., Abramoff, M., Niemeijer, M., Viergever, M., van Ginneken, B.: Ridgebased vessel segmentation in color images of the retina. IEEE Transactions on Medical Imaging 23, 501-509 (2004)

16. Tsai, C., Stewart, C., Tanenbaum, H., Roysam, B.: Model-based method for improving the accuracy and repeatability of estimating vascular bifurcations and crossovers from retinal fundus images. IEEE Transactions on Information Technology in Biomedicine 8, 122-130 (2004)

17. Tso, M., Jampol, L.: Path-physiology of hypertensive retinopathy. Opthalmology 89 (1982) 